\title{
Equation of state and elasticity of brucite at high temperatures and pressures from classical atomistic simulations
}

EVGENY V. TARARUSHKIN ${ }^{1,2}$ AND ANDREY G. KALINICHEV ${ }^{1,3}$

${ }^{1}$ National Research University Higher School of Economics

${ }^{2}$ HSE University

${ }^{3}$ IMT Atlantique

Presenting Author: etararushkin@hse.ru

The behavior of brucite, $\mathrm{Mg}(\mathrm{OH})_{2}$, over wide ranges of temperature and pressure is of great interest for fundamental geophysics because this mineral itself plays a major role in equilibria controlling water balance in the mantle's subduction zones, and because the octahedral $\mathrm{Mg}(\mathrm{OH})_{6}$ structural units and brucite-type layers also constitute an important part of layered dense magnesium hydrous silicates, DMHS - other important players in the subduction zone water balance.

Brucite has a simple hexagonal layered crystal structure that is held together by hydrogen bonds between the hydroxyl groups of the opposing layers. Its properties have already been well studied by various methods of classical atomistic simulations and DFT methods. Here we are using the new recent modification of the classical force field, ClayFF-MOH [1], which accurately accounts for the bending of $\mathrm{Mg}-\mathrm{O}-\mathrm{H}$ angles in the brucite structure. ClayFF has been originally developed for atomistic computer simulations of clay-related minerals and their hydrated interfaces under ambient conditions [2], and the crystallographic parameters of brucite at $25^{\circ} \mathrm{C}$ and 1 bar were used, among several others, to develop that earlier parametrization. Now we are testing the applicability of the new more accurate ClayFF$\mathrm{MOH}$ model over very wide ranges of temperature and pressure up to $400^{\circ} \mathrm{C}$ and $150 \mathrm{kbar}$, well beyond the range of its original parametrization.

The pressure and temperature dependencies of brucite compressibility, the elastic properties, the coefficients of thermal expansion, and the vibrational spectra are calculated in a series of classical molecular dynamics simulations using the ClayFF-MOH model. The results demonstrate that ClayFF$\mathrm{MOH}$, as simple and approximate as is, can be quite accurate in predicting mineral properties at subduction zone conditions, which greatly expands the area of applicability for this force field model.

The research was conducted within the framework of the HSE University Basic Research Program. The calculations were performed on the CHARISMa supercomputer of NRU HSE.

\section{References}

[1] Pouvreau M., Greathouse J. A., Cygan R. T., Kalinichev A. G. (2017) J. Phys. Chem. C 121, 14757-14771.

[2] Cygan R.T., Liang J.-J., Kalinichev A. G. (2004) J. Phys. Chem. B 108, 1255-1266. 